Pacific Journal of Mathematics

SYMPTOTICS FOR CERTAIN WIENER INTEGRALS

RICHARD J. GRIEGO AND ANDRZEJ KoRZENIOWS 


\section{ASYMPTOTICS FOR CERTAIN WIENER INTEGRALS ASSOCIATED WITH HIGHER ORDER DIFFERENTIAL OPERATORS}

\section{RICHARD J. GRIEGO AND ANDRZEJ KORZENIOWSKI}

The aim of this paper is to derive a large deviation principle for a certain class of higher order operators by combining the ideas of Donsker and Varadhan with the random evolution point of view of Griego and Hersh.

1. Introduction. It has been known for some time how to recover the principal eigenvalue for operators that generate Markov semigroups by means of the Large Deviation Principle of Donsker and Varadhan ([3], [4], [5]). The principal eigenvalues for such operators will be obtained as limits of certain functionals of Brownian motion.

We shall consider operators of the form $L=\frac{1}{2} \Delta_{x}+c(x) A_{y}$, where the Laplacian $\Delta_{x}$ is stochastic in the sense that it generates the Brownian motion semigroup; whereas $A_{y}$ is analytic and does not correspond in general to a Markov process. The operator $L$ can be interpreted as either the averaged result of randomization of the evolutions $c(x) A_{y}$ driven through the variable $x$ in the coefficient $c(x)$ by Brownian motion or as a perturbation of the Laplacian by an operator-valued potential $V(x)=c(x) A_{y}$.

We follow the notation of [7], and we will recall some necessary facts. Let $A_{y}=\sum_{|\alpha| \leq 2 r} a_{\alpha}(y) D^{\alpha}$ be a formally self-adjoint elliptic operator of order $2 r$ on a bounded open set $G \subset R^{m}$, with domain $D\left(A_{y}\right)$ being a subset of the Sobolev space $H_{2 r}(G)$, such that $\left(A_{y} g, g\right) \leq$ 0 for $g \in D\left(A_{y}\right)$ with the inner product of $L^{2}(G)$.

In what follows we shall consider the following initial-boundary value problem

$$
\begin{aligned}
& u_{t}\left(y, y^{\prime}, t\right)=A_{y} u, \quad t>0, y, y^{\prime} \in G \\
& u\left(y, y^{\prime}, 0\right)=\delta\left(y-y^{\prime}\right)
\end{aligned}
$$

subject to given homogeneous conditions on the boundary $\partial G$. We shall assume that the (fundamental) solution to this problem can be 
written as follows:

$$
u\left(y, y^{\prime}, t\right)=\sum_{n=1}^{\infty} e^{-\alpha_{n} t} \phi_{n}(y) \phi_{n}\left(y^{\prime}\right), \quad \cdots \leq-\alpha_{2} \leq-\alpha_{1}<0
$$

where $\left\{\phi_{n}\right\}$ are complete and orthonormal eigenfunctions of $A_{y}$ with corresponding eigenvalues $\left\{-\alpha_{n}\right\}$. We assume the multiplicity of $\alpha_{1}$ is $k$.

As shown by Gårding [6], this assumption is satisfied whenever Dirichlet boundary conditions are imposed, i.e., $\partial^{j} u / \partial \nu^{j}=0$ for $j=0,1, \ldots, r-1$, where $\partial / \partial \nu$ is differentiation with respect to the outward normal to $G$.

Let $c: R^{n} \rightarrow[0, \infty)$ be a locally Hölder function that approaches infinity for large $x$, i.e.,

$$
\begin{aligned}
& c(x) \rightarrow \infty, \quad \text { as }|x| \rightarrow \infty \quad \text { and } \\
& \left|c(x)-c\left(x^{\prime}\right)\right|<M(x)\left|x-x^{\prime}\right|^{\alpha}, \quad 0<\alpha<1
\end{aligned}
$$

for $x^{\prime}$ in some neighborhood of $x$ for every $x$ in $R$.

Then, by Lemma 2 of [7], $L=\frac{1}{2} \Delta_{x}+c(x) A_{y}$ acting on $\psi(x, y) \in$ $L^{2}\left(R^{n} \times G\right)$ with $\psi(\cdot, y) \in D\left(\Delta_{x}\right)$ and $\psi(x, \cdot) \in D\left(A_{y}\right)$ itself has negative eigenvalues $\left\{-\lambda_{n}\right\}, \cdots-\lambda_{2} \leq-\lambda_{1}<0$, with a complete set of orthonormal eigenfunctions $\psi_{n}(x, y) \in L^{2}\left(R^{n} \times G\right)$. See also below for a direct derivation of these facts.

We will consider functionals $\Phi$ satisfying the conditions of [3]. Thus, let $\mathscr{F}$ be the space of probability distribution functions on $R^{n}$ with the Levy metric. Let $\Phi: \mathscr{F} \rightarrow[0, \infty]$ be a function such that (a) $\Phi$ is lower semicontinuous on $\mathscr{F}$; (b) $\Phi^{-1}((-\infty, K])$ is compact in $\mathscr{F}$ for each $0<K<\infty$; (c) if $F_{m} \rightarrow F$ and supp $F_{m} \subset\left[a_{1}, b_{1}\right] \times \cdots \times\left[a_{n}, b_{n}\right]$, then $\Phi\left(F_{m}\right) \rightarrow \Phi(F)$; (d) let $F \in \mathscr{F}$ with $\Phi(F)<\infty$ and let $g_{m}: R^{n} \rightarrow$ $[0,1]$ be continuous with $g_{m}(x)=1$ for $x \in[-n, n] \times \cdots \times[-n, n]$. If $F_{m}$ is defined by

$$
f_{m}(x)=\frac{\int_{-\infty}^{x_{1}} \cdots \int_{-\infty}^{x_{n}} g_{m}(y) d F(y)}{\int \cdots \int_{R^{n}} g_{m}(y) d F(y)} ; \quad \text { where } x=\left(x_{1}, \ldots, x_{n}\right),
$$

then $\Phi\left(F_{m}\right) \rightarrow \Phi(F)$.

If $F$ has density $f$, we write $\Phi(f)$ for $\Phi(F)$.

Let $\Omega$ be the space of continuous functions $\omega:[0, \infty) \rightarrow R^{n}$ and let $X(s, \omega)=\omega(s)$. Define for $B \subset R^{n}$,

$$
L(t, \omega, B)=(1 / t) \int_{0}^{t} I_{B}(X(s, \omega)) d s,
$$


which is the proportion of time in $[0, t]$ that the path $X(\cdot, \omega)$ spends in the set $B$. Also, let $E_{x}$ denote expectation with respect to Wiener measure $P_{x}$ on $\Omega$, so that $P_{x}(\omega: X(0, \omega)=x)=1$.

Finally, let $\mathscr{F}^{+}=\left\{f \in L^{2}\left(R^{n}\right) \cap C^{1}\left(R^{n}\right):\|f\|_{1}=1\right.$ and $f>0$ on $R^{n}$ or on Interior(supp $\left.(f)\right)$ if $\operatorname{supp}(f)$ is compact $\}$ and let $\mathscr{G}=$ $\left\{g \in L^{2}(G) \cap D\left(A_{y}\right):\|g\|_{2}=1\right\}$.

Under the above conditions on the functional $\Phi$, Donsker and Varadhan [3, Theorem 2.1] have proved the following asymptotic evaluation.

THEOREM (Donsker-Varadhan). For each $x$ in $R^{n}$,

$$
\begin{aligned}
\lim _{t \rightarrow \infty} & \frac{1}{t} \log E_{x}\left[e^{-t \Phi(L(t, \omega, \cdot))}\right] \\
& =-\inf \left\{\frac{1}{8} \int_{R^{n}} \frac{|\nabla f(x)|^{2}}{f(x)} d x+\Phi(f): f \in \mathscr{F}^{+}\right\} .
\end{aligned}
$$

The following corollary follows by considering

$$
\Phi(f)=\int V(x) f(x) d x
$$

COROLlary. If $V \geq 0$ is continuous and $V(x) \rightarrow \infty$ as $|x| \rightarrow \infty$, then the principal eigenvalue $-\lambda_{1}$ of the operator $\frac{1}{2} \Delta_{x}-V(x)$ on $L^{2}\left(R^{n}\right)$ is given by

$$
\begin{aligned}
-\lambda_{1}=\lim _{t \rightarrow \infty} \frac{1}{t} \log E_{x}\left[\exp \left(-\int_{0}^{t} V(X(x)) d s\right)\right] \\
=-\inf \left\{\frac{1}{8} \int_{R^{n}} \frac{|\nabla f(x)|^{2}}{f(x)} d x+\int_{R^{n}} V(x) f(x) d x: f \in \mathscr{F}^{+}\right\} \\
=-\inf \left\{\frac{1}{2} \int_{R^{n}}|\nabla \psi(x)|^{2} d x\right. \\
\left.+\int_{R^{n}} V(x) \psi^{2}(x) d x: \psi \in L^{2}\left(R^{n}\right),\|\psi\|_{2}=1\right\} .
\end{aligned}
$$

These results appear in [3] for $n=1$, but they readily carry over to higher dimensions by mimicking the proofs there.

The main result of this paper is the following version of the DonskerVaradhan theorem in our setup. 
MAIN Theorem. Let $u\left(y, y^{\prime}, t\right)$ be as in (I.1). Then,

$$
\begin{aligned}
\lim _{t \rightarrow \infty} \frac{1}{t} \log E_{x}\left[\int_{G} u(y, y, t \Phi(L(t, \omega, \cdot))) d y\right] \\
=-\inf _{f} \inf _{g}\left\{\frac{1}{8} \int_{R^{n}} \frac{|\nabla f(x)|^{2}}{f(x)} d x\right. \\
\left.\quad+\left(-A_{y} g, g\right) \Phi(f): f \in \mathscr{F}^{+}, g \in \mathscr{G}\right\} .
\end{aligned}
$$

COROLlaRY. The principal eigenvalue $-\lambda_{1}$ of $L=1 / 2 \Delta_{x}+c(x) A_{y}$ on $L^{2}\left(R^{n} \times G\right)$ is given by

$$
\begin{aligned}
-\lambda_{1}=\lim _{t \rightarrow \infty} \frac{1}{t} \log E_{x}\left[\int_{G} u(y, y, \alpha(t)) d y\right] \\
=-\inf _{f} \inf _{g}\left\{\frac{1}{8} \int_{R^{n}} \frac{|\nabla f(x)|^{2}}{f(x)} d x\right. \\
\left.+\left(-A_{y} g, g\right) \int_{R^{n}} c(x) f(x) d x: f \in \mathscr{F}^{+}, g \in \mathscr{G}\right\} \\
=-\inf _{\psi} \inf _{g}\left\{\frac{1}{2} \int_{R^{n}}|\nabla \psi(x)|^{2} d x\right. \\
+\left(-A_{y} g, g\right) \int_{R^{n}} c(x) \psi^{2}(x) d x: \\
g\left(G \in \mathscr{G}, \psi \in L^{2}\left(R^{n}\right),\|\psi\|_{2}=1\right\}
\end{aligned}
$$

where $\alpha(t)=\int_{0}^{t} c(x(s)) d s$.

Observe that when $A_{y} \equiv-1$, then $u\left(y, y^{\prime}, t\right)=e^{-t} \delta\left(y-y^{\prime}\right)$ and we recover (I.4).

Proofs are given in the next section.

EXAMPLES. As a simple illustration, consider the fourth order operator

$$
L=\frac{1}{2} \frac{\partial^{2}}{\partial x^{2}}-c(x) \frac{\partial^{4}}{\partial y^{4}}, \quad \text { for }(x, y) \in R^{1} \times(0,1),
$$

which arises in elasticity theory. We impose boundary conditions $g(0)=g(1)=g^{\prime \prime}(0)=g^{\prime \prime}(1)=0$ on $A_{y}$. Then $\left(-A_{y} g, g\right)=\left(g^{\prime \prime}, g^{\prime \prime}\right)$ and we obtain

$$
\lambda_{1}=\inf \left\{\frac{1}{8} \int_{-\infty}^{\infty} \frac{\left(f^{\prime}(x)\right)^{2}}{f(x)} d x+\pi^{4} \int_{-\infty}^{\infty} c(x) f(x) d x: f \in \mathscr{F}^{+}\right\} .
$$


Since $\alpha_{1}=\inf \left\{\left(g^{\prime \prime}, g^{\prime \prime}\right): g \in \mathscr{G}\right\}=\pi^{4}$ with corresponding eigenfunctions $\phi(y)=\sin \pi y$. See [1, p. 146] for other examples of possible boundary conditions.

Another example is $A_{y}=-\Delta^{2}$ with $u=0$ and $\partial u / \partial \nu=0$ on $\partial G$ in $R^{2}$ that corresponds to a vibrating plate; see [2, p. 460].

\section{Proofs.}

Lemma. Let $0<p<\infty$ and $0<\varepsilon<a<\infty$ be given. Then for real numbers such that $0<\alpha_{1}<\alpha_{2} \leq \cdots$ and $\alpha_{n} \sim C n^{p}$ as $n \rightarrow \infty$, there is a constant $M=M\left(\alpha_{1}, p, C, \varepsilon\right)$ such that $\sum_{n=2}^{\infty} e^{-\alpha_{n} a} \leq M e^{-\alpha_{1} a}$.

Proof. Let $b=C / 2 \alpha_{1}$. Then by the assumption there is an $N$ such that $\alpha_{n} \geq \alpha_{1} b n^{p}$ and $b N^{p} \geq 1$ for $n \geq N$. Hence, $\sum_{n=2}^{\infty} e^{-\alpha_{n} a} \leq$ $N e^{-\alpha_{1} a}+\sum_{n=N+1}^{\infty} e^{-\alpha_{n} a}$ and the second term on the right is dominated by

$$
\begin{aligned}
\int_{N}^{\infty} e^{-\alpha_{1} a b x^{p}} d x & =\frac{e^{-\alpha_{1} a}}{p b^{1 / p}} \int_{b N^{p}}^{\infty} e^{-\alpha_{1} a(z-1)} z^{(1-p) / p} d z \\
& \leq e^{-\alpha_{1} a}\left[\frac{1}{p b^{1 / p}} \int_{0}^{\infty} e^{-\alpha_{1} \varepsilon z}(1+z)^{(1-p) / p} d z\right] .
\end{aligned}
$$

Letting $M$ be the finite constant in the brackets we otain the result of the lemma.

We also need some information about the eigenvalues $\left\{-\alpha_{n}\right\}$ of $-A_{y}$ in the form of a Weyl-type theorem. First, define $w(y)=$ $m\left(\xi: 0<a^{0}(y, \xi)<1\right)$ where $m$ is Lebesgue measure and $W(G)=$ $\int_{G} w(y) d y$, where $a^{0}(y, \xi)=\sum_{|\alpha|=2 r} a_{\alpha}(y) \xi^{\alpha}$. Gårding has shown the following asymptotic expression for the eigenvalues:

$$
N(\alpha)=\sum_{\alpha_{n} \leq \alpha} 1 \sim(2 \pi)^{-m} W(G) \alpha^{m / 2 r} \quad \text { as } \alpha \rightarrow \infty \text { (see [6, p. 239]). }
$$

Since $N(\alpha)=n$ for $\alpha=\alpha_{n}$ we have that

$$
\alpha_{n} \sim C n^{p} \quad \text { as } n \rightarrow \infty
$$

with $p=2 r / m$ and $C=(2 \pi)^{2 r} W(G)^{-p}$.

Comparing this with our examples, we have in the case $A_{y}=$ $-\partial^{4} / \partial y^{4}$ that $\alpha_{n}=\pi^{4} n^{4}$ and for $A_{y}=-\Delta^{2}$ we obtain

$$
\alpha_{n} \sim\left(\frac{4 \pi}{\text { area of the plate }}\right)^{2} n^{2} .
$$

Both of these results agree with (II.1). 
Returning to the proof of the Main Theorem, in order to show (I.5) put $\alpha=\Phi(L(t, \omega, \cdot))$ and assume for now that $a \geq \varepsilon>0$. Then by (I.2) we have

$$
u(y, y, a t)=\sum_{n=1}^{\infty} e^{-\alpha_{n} a t} \phi_{n}^{2}(y) .
$$

Integrating with respect to $y$ in this expression we obtain by orthonormality of the $\phi_{n}$ 's that

$$
\int_{G} u(y, y, a t) d y=\sum_{n=1}^{\infty} e^{-\alpha_{n} a t}
$$

Applying the Lemma and (II.1) we get

$$
k e^{-\alpha_{1} a t} \leq \sum_{n=1}^{\infty} e^{-\alpha_{n} a t} \leq e^{-\alpha_{1} a t}(k+M)
$$

where $k$ is the multiplicity of $\alpha_{1}$ and the constant $M$ does not depend on the sample path $\omega$. Taking $E_{x}$ and then $(1 / t) \log$ of both sides of (II.2) and letting $t \rightarrow \infty$ we obtain

$$
\begin{aligned}
\lim _{t \rightarrow \infty} & \frac{1}{t} \log E_{x}\left[\int_{G} u(y, y, t \Phi(L(t, \omega, \cdot)))\right] \\
& =\lim _{t \rightarrow \infty} \frac{1}{t} \log E_{x}\left[e^{-\alpha_{1} t \Phi(L(t, \omega, \cdot))}\right] \\
& =-\inf _{f \in \mathscr{F}^{+}}\left\{\frac{1}{8} \int_{G} \frac{|\nabla f(x)|^{2}}{f(x)} d x+\alpha_{1} \Phi(f)\right\}
\end{aligned}
$$

where the second equality comes from the Donsker-Varadhan result (I.3).

This proves (I.5) for $\Phi \geq \varepsilon>0$ because $\alpha_{1}=\inf _{g \in \mathscr{G}}\left(-A_{y} g, g\right)$. To see that the $\varepsilon$ condition is immaterial, apply (II.3) for $\tilde{\Phi}=\Phi+\varepsilon$ then $-\alpha_{1} \varepsilon$ cancels on all sides of (II.3) proving (I.5) for all functionals satisfying the conditions of Donsker-Varadhan as required by the Main Theorems. This proves the Main Theorem.

Turning to the proof of the Corollary and (I.6), the way that one obtains the eigenvalues and eigenfunctions for $L=\frac{1}{2} \Delta_{x}+c(x) A_{y}$ in [7] is a two-stage process that leads to a double index for both eigenvalues and eigenfunctions as follows. Fix $n$ and consider the operator $\frac{1}{2} \Delta_{x}-\alpha_{n} c(x)$ operating on $L^{2}\left(R^{n}\right)$. By the conditions on $c(x)$ and a result of Ray [8, Theorem 3], this operator has negative eigenvalues $\left\{-\beta_{m, n}, m=1,2, \ldots\right\}$ and complete orthonormal eigenfunctions 
$\left\{u_{m, n}(x), m=1,2, \ldots\right\}$ in $L^{2}\left(R^{n}\right)$. This implies

$$
\begin{aligned}
L\left(u_{m, n}(x) \phi_{n}(y)\right) & =\frac{1}{2} \Delta_{x} u_{m, n}(x) \phi_{n}(y)+c(x) A_{y} u_{m, n}(x) \phi_{n}(y) \\
& =\left(\frac{1}{2} \Delta_{x}-\alpha_{n} c(x)\right) u_{m, n}(x) \phi_{n}(y) \\
& =-\beta_{m, n} u_{m, n}(x) \phi_{n}(y) .
\end{aligned}
$$

Hence, the $-\beta_{m, n}$ for $m, n=1,2, \ldots$, are eigenvalues of $L=\frac{1}{2} \Delta_{x}+$ $c(x) A_{y}$ with corresponding eigenfunctions $u_{m, n}(x) \phi_{n}(y)$.

Now, by the Feynman-Kac formula, if the largest eigenvalue of $\frac{1}{2} \Delta_{x}-\alpha c(x)$ is $\lambda(\alpha)$, then

$$
\lambda(\alpha)=\lim _{t \rightarrow \infty} \frac{1}{t} \log E_{x}\left[\exp \left(-\alpha \int_{0}^{t} c(X(s)) d s\right)\right]
$$

implying that $\lambda(\alpha)$ decreases as $\alpha$ increases, which in turn implies $-\beta_{1,1}=\sup _{m, n}\left(-\beta_{m, n}\right)$, i.e., the supremum is itself an eigenvalue. We label the eigenvalues in a linear array $\cdots \leq-\lambda_{2} \leq-\lambda_{1}<0$, so that $-\lambda_{1}=-\beta_{1,1}$.

By (I.4) we have that

$$
\begin{aligned}
-\lambda_{1}= & \lim _{t \rightarrow \infty} \frac{1}{t} \log E_{x}\left[\exp \left(-\alpha_{1} \int_{0}^{t} c(X(s)) d s\right)\right] \\
=-\inf \{ & \left\{\frac{1}{8} \int_{R^{n}} \frac{|\nabla f(x)|^{2}}{f(x)} d x+\alpha_{1} \int_{R^{n}} c(x) f(x) d x: f \in \mathscr{F}^{+}\right\} \\
=-\inf \{ & \left\{\frac{1}{2} \int_{R^{n}}|\nabla \psi(x)|^{2} d x\right. \\
& \left.+\alpha_{1} \int_{R^{n}} c(x) \psi^{2}(x) d x: \psi \in L^{2}\left(R^{n}\right),\|\psi\|_{2}=1\right\} .
\end{aligned}
$$

For $\Phi(f)=\int c(x) f(x) d x$ we have that

$$
t \Phi(L(t, \omega, \cdot))=\int_{0}^{t} c(X(s)) d s=\alpha(t)
$$

thus by (II.3) and the fact that $\alpha_{1}=\inf _{g \in \mathscr{G}}\left(-A_{y} g, g\right)$ we obtain (I.6) upon substitution into (II.4), there by proving our corollary.

REMARK. Under an additional weak condition on the operator $A_{y}$, it is possible to show for each $y_{0} \in G$ that

$$
\begin{aligned}
\lim _{t \rightarrow \infty} & \frac{1}{t} \log \left|E_{x}\left[\int_{G} u\left(y_{0}, y, t \Phi(L(t, \omega, \cdot))\right) d y\right]\right| \\
& =\lim _{t \rightarrow \infty} \frac{1}{t} \log E_{x}\left[\int_{G} u(y, y, t \Phi(L(t, \omega, \cdot))) d y\right] .
\end{aligned}
$$


Note that the absolute value on the left-hand side is necessary since $u\left(y_{0}, y, t\right)$ is not nonnegative in general.

Our results can be extended to operators of the form $L=D+$ $V(x)$ where $D$ is the generator of a wide class of Markov processes satisfying the assumptions of the Donsker-Varadhan theory and for fairly general operator-valued potentials $V(x)$ treated in the theory of random evolutions.

\section{REFERENCES}

[1] L. Collatz, Eigenwerte Probleme, Chelsea, New York, 1948.

[2] R. Courant and D. Hilbert, Methods of Mathematical Physics, Vol. 1, Interscience-Wiley, New York, 1953.

[3] M. D. Donsker and S. R. S. Varadhan, Asymptotic evaluation of certain Wiener integrals for large time, Proceedings of International Conference of Function Space Integration, Oxford, (1974), 15-33.

[4] - Asymptotic evaluation of certain Markov process expectations for large time I, Comm. Pure Appl. Math., 28 (1975), 1-47.

[5] _-, Asymptotics for the Wiener sausage, Comm. Pure Appl. Math., 28 (1975), 525-565.

[6] L. Gårding, On the asymptotic distribution of the eigenvalues and eigenfunctions of elliptic differential operators, Math. Scand., 1 (1953), 237-255.

[7] R. J. Griego and R. Hersh, Weyl's theorem for certain operator-valued potentials, Indiana Univ. Math. J., 27 (1978), 195-209.

[8] D. Ray, On spectra of second-order differential operators, Trans. Amer. Math. Soc., 77 (1954), 299-321.

Received May 2, 1988.

UNiversity OF New MeXico

ALBUQUERQUE, NM 87131

AND

UNIVERSITY OF TEXAS

ARLINGTON, TX 76019 


\section{PACIFIC JOURNAL OF MATHEMATICS EDITORS}

\author{
V. S. VARADARAJAN \\ (Managing Editor) \\ University of California \\ Los Angeles, CA 90024-1555-05 \\ Herbert Clemens \\ University of Utah \\ Salt Lake City, UT 84112 \\ ThOMAS ENRIGHT \\ University of California, San Diego \\ La Jolla, CA 92093
}

\section{R. FINN}

Stanford University

Stanford, CA 94305

HeRmann FlaschKa

University of Arizona

Tucson, AZ 85721

VAUGHAN F. R. JONES

University of California

Berkeley, CA 94720

Steven KerckhofF

Stanford University

Stanford, CA 94305

\author{
RobION KIRBY \\ University of California \\ Berkeley, CA 94720 \\ C. C. Moore \\ University of California \\ Berkeley, CA 94720 \\ HAROLD STARK \\ University of California, San Diego \\ La Jolla, CA 92093
}

\section{ASSOCIATE EDITORS}
R. ARENS
E. F. BECKenBach
B. H. NeumanN
F. WOLF
K. YoshidA (1906-1982)
(1904-1989)

\section{SUPPORTING INSTITUTIONS}

UNIVERSITY OF ARIZONA

UNIVERSITY OF BRITISH COLUMBIA

CALIFORNIA INSTITUTE OF TECHNOLOGY

UNIVERSITY OF CALIFORNIA

MONTANA STATE UNIVERSITY

UNIVERSITY OF NEVADA, RENO

NEW MEXICO STATE UNIVERSITY

OREGON STATE UNIVERSITY
UNIVERSITY OF OREGON

UNIVERSITY OF SOUTHERN CALIFORNIA

STANFORD UNIVERSITY

UNIVERSITY OF HAWAII

UNIVERSITY OF TOKYO

UNIVERSITY OF UTAH

WASHINGTON STATE UNIVERSITY

UNIVERSITY OF WASHINGTON 


\section{Pacific Journal of Mathematics}

Vol. 142, No. $1 \quad$ January, 1990

Marco Andreatta, Mauro Beltrametti and Andrew Sommese, Generic properties of the adjuction mapping for singular surfaces and applications

Chen-Lian Chuang and Pjek-Hwee Lee, On regular subdirect products of

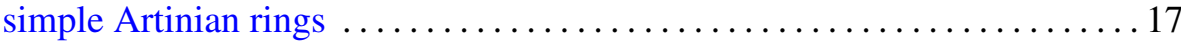

Fernando Giménez and Vicente Miquel Molina, Volume estimates for real hypersurfaces of a Kaehler manifold with strictly positive holomorphic sectional and antiholomorphic Ricci curvatures $\ldots \ldots \ldots \ldots \ldots \ldots 23$

Richard J. Griego and Andrzej Korzeniowski, Asymptotics for certain Wiener integrals associated with higher order differential operators

Abdeslam Mesnaoui, Unitary bordism of classifying spaces of quaternion

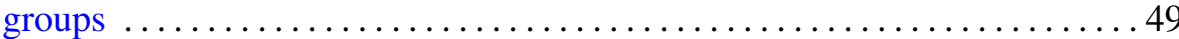

Abdeslam Mesnaoui, Unitary cobordism of classifying spaces of quaternion

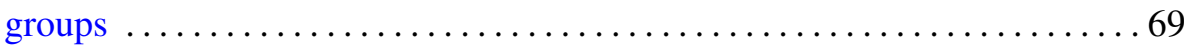

Jesper M. Møller, On equivariant function spaces $\ldots \ldots \ldots \ldots \ldots \ldots \ldots \ldots$

Bassam Nassrallah, A $q$-analogue of Appell's $F_{1}$ function, its integral representation and transformations

Peter A Ohring, Solvability of invariant differential operators on metabelian

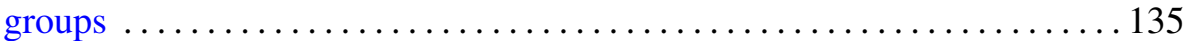

Athanase Papadopoulos and R. C. Penner, Enumerating pseudo-Anosov foliations

Ti-Jun Xiao and Liang Jin, On complete second order linear differential

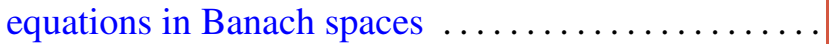

Carl Widland and Robert F. Lax, Weierstrass points on Gorenstein curves 